\title{
Therapeutic targets of type 2 diabetes: an overview
}

\begin{abstract}
As the prevalence of type 2 diabetes (T2D) is rapidly increasing, and effective strategies to manage and prevent this disease are urgently required, as per WHO statistics undertaken in 2012, the number of people suffering from T2D in India is expected to shoot up from 40.9 million in 2007 to 69.9 million by the year 2025 . Even though, the symptoms of T2D are seen in the later stages of life, the onset of the disease occurs quite early and is referred to as lifestyle disorder. Insulin resistance and $\beta$ cell dysfunction are the main causative factors along with mutations in the genes regulating glucose homeostasis and $\beta$ cell development. A vicious circle of the causes and effects of hyperglycemia interlinked together forms the whole picture of T2D. Our review discusses the molecular targets of the current oral therapeutic drugs, which cuts down hyperglycemia. This review aims to provide better insights to comprehension of the disease, recent advances in molecular and cellular therapies. A comprehensive analysis of these novel targets identified over the years and various therapeutic leads which successfully completed their clinical trials are presented in this review. Few of these lead molecules operate as GLP-1 agonist, SGLT2 inhibitors, and DPP-IV inhibitors and are termed as next generation anti-diabetic drugs.
\end{abstract}

Keywords: therapeutic targets, type 2 diabetes, target genes, tumor necrosis factor alpha
Volume I Issue 3 - 2017

\author{
Ananya Bhowmick and Sofia Banu \\ Department of Bioengineering and Technology, Gauhati \\ University Assam, India
}

\begin{abstract}
Correspondence: Sofia Banu, Department of Bioengineering and Technology, GUIST, Gauhati University, Assam-78I0I4, Tel
\end{abstract} 8811078986,Email sofiabanu2@gmail.com

Received: May 05, 2017 | Published: July 13, 2017
Abbreviations: T1D, type 1 diabetes; T2D, type 2 diabetes; NIDDM, non-insulin-dependent diabetes mellitus; TNF- $\alpha$, tumor necrosis factor alpha; IRS, insulin receptor and substrates; RXR, retinoid X-receptor; PTP1B, protein tyrosine phosphatase 1B; PTPs, protein tyrosine phosphatases; GPR, G-protein coupled receptor; LA, lipoic acid; NAC, N-acetyl-l-cysteine; SUR, sulfonylurea receptor; SGLT, sodium glucose transporter inhibitor; HESCs, human embryonic stem cells; FDA, food and drug administration

\section{General introduction}

Diabetes as a chronic disorder where the pancreas is unable to synthesize enough insulin and the body also develops resistance to the insulin it produces under normal physiologic conditions. As per the Current International Diabetes Federation and WHO reports, a surge from 382 million people in 2013 to 592 million by 2035 is expected to occur. ${ }^{1,2}$ WHO have recognized two major types of diabetes: Type 1 diabetes (T1D) and Type 2 diabetes (T2D). T1D is caused as an effect of insufficient insulin production by the pancreatic $\beta$-cells, whereas T2D develop due to insulin insensitivity in the targeted tissues. ${ }^{3}$ In T2D patients develop resistance to secreted insulin and can be treated with lifestyle modifications like diet, regular exercise and medication. Among all diabetic cases, $90-95 \%$ suffers from T2D, the most prevalent form of diabetes. ${ }^{4}$ The characteristic symptoms of T2D are elevated sugar levels in blood, unusual thirst, extreme tiredness, sudden hunger pangs, polyuria, polyphagia, random weight loss, and other factors including dislipidemia that further leads to the development of micro and macrovascular complications, finally causing morbidity. ${ }^{5}$ Researchers from various disciplines are trying to develop safer and effective therapeutic approaches by meticulous evaluation of the effectiveness of natural and synthetically derived lead molecules on different novel protein targets. Modern day drugs for diabetes include sulfonylureas, GLP-1 agonist, PPAR- $\gamma$ agonists,
DPP4 inhibitors, metformin, rosiglitazone, etc. and some very recent ones like SGLT2 inhibitors. In this review, we have discussed few of the mostly researched upon promising target genes and lead molecules having the potential to be established as a safe and effective next generation anti-diabetic agent.

\section{Diabetes complications and therapeutic targets}

WHO assumes that diabetes will be ranked as the $7^{\text {th }}$ leading cause of fatality in 2030? Type 2 is also referred to as non-insulindependent diabetes mellitus (NIDDM), and occurs largely in elderly group of people especially who are overweight and have a sedentary lifestyle. High incidence of occurrence of T2D has been observed in African, Americans, Native of Hawaiians, Indians and Hispanics/ Latinos. Within the last two decades, T2D has affected more than 300 million people across the globe, mainly covering China, India and United States of America. ${ }^{6,7}$ The normal rate of hepatic glucose production increases in T2D patients, resulting in decreased insulin secretion (due to reduced number of $\beta$ cells), increased glucagon secretion and hepatic insensitivity to insulin. Collective effect of all these phenomenons damages the healthy glucose uptake of adjacent tissues. ${ }^{89}$ Several factors regulate receptor mediated insulin resistance mechanism. Few of the significant factors include elevated serine/ threonine phosphorylation of the insulin receptor, deactivation of the receptor, decreased tyrosine phosphorylation, autoantibody formation against the receptor and inherited structural abnormality of the receptor. Disruption of insulin action also arises at post-receptor events, chiefly glucose transport. ${ }^{10}$ There are a number of different hypotheses explaining the mechanism of insulin resistance in obesity. It was proposed that tumor necrosis factor alpha (TNF- $\alpha$ ) is released by adipose tissue and is able to impair insulin signaling through serine kinase and tyrosine phosphatase dependent modulation of the insulin signaling chain at the level of the insulin receptor and substrates (IRS). ${ }^{11}$ 
Diabetes is also renowned as an oxidative stress disorder caused by inequity between free radical formation in the body and the capacity of the body's natural antioxidant system to scavenge them. Hyperglycemia is the main causative factor behind the diabetes associated critical complications including obesity, nephropathy, retinopathy, vascular damage and neuropathy. ${ }^{12,13}$ Oxidative stress caused by increased ROS production plays a key role in the development of diabetes related physiological complications. In diabetic patients, the level of superoxide dismutase (enzyme which neutralizes the superoxide radical), catalase (enzyme enagaed for removal of $\mathrm{H}_{2} \mathrm{O}_{2}$ ), are extensively reduced. ${ }^{14,15}$ Hence this accumulated oxidative stress activates multiple stress sensitive signaling pathways, resulting in cellular damage, damage of proteins, lipids, and DNA. ${ }^{16,17}$ Thus, several initiatives have been taken to cut down oxidative stress in patients with diabetes, by administration of naturally occurring anti-oxidants like vitamin $\mathrm{E}$, vitamin $\mathrm{C}$, and lipoic acid. ${ }^{18}$ In our review we will discuss about how antioxidants serve as potential molecules in preventing activation of stress pathways under hyperglycaemic conditions. Elucidating the molecular basis of the mode of action of these anti-oxidants could guide us to the detection of novel pharmacological targets and lead molecules for combating the ill-effect of diabetes.

\section{The mostly targeted genes and pathways are}

Peroxisome proliferator-activated receptor-gamma (PPAR- $\gamma$ )PPAR- $\gamma$ is a nuclear receptor, highly expressed in the fatty tissues and in skeletal muscle cells. Its cellular levels are modulated by insulin resistance, glucocorticoids and cytokines like the tumor necrosis factor. ${ }^{19}$ The PPAR- $\gamma$ gene functions by facilitating insulin sensitivity by its agonist in muscle cells, to enable maximum glucose uptake in adipose tissues which maintain body homeostasis. PPAR- $\gamma$ when attached with an agonist (thiazolidinedione), gets activated by forming complex with another transcription factor named retinoid X-receptor (RXR), and finally binds to a specific DNA motif (peroxisome proliferate response element) in the promoters region of target gene. ${ }^{20}$ Thiazolidinedione (glitazones) mainly act on PPAR- $\gamma$ and stimulates insulin sensitivity.

Protein tyrosine phosphatase 1B (PTP1B)-The function of Protein tyrosine phosphatases (PTPs) enzyme is to control tyrosine phosphorylation in cells. Tyrosine phosphorylation in the insulinreceptor activation pathway leads to insulin signal transduction and PTPs removes phosphate groups from phosphorylated tyrosine residues on proteins. ${ }^{21}$ PTP1B has evolved as a recent target for drug design against T2D and obesity, primarily because inhibition of PTP1B significantly cuts down storage of triglycerides in adipose tissues under presence of excess nutrients supply. Thus, PTP1B inhibitors might serve as a novel type of insulin activator and aid in the management of T2D, obesity and related cardiovascular ailments. ${ }^{22}$

G-protein coupled receptor (GPR)-GPR120 is a member of rhodhopsin family of $\mathrm{G}$ protein coupled receptors which is profoundly expressed in the intestinal tract and adipose tissue cells. GPR120 activation stimulates glucagon like peptide 1 (GLP-1) secretion and associated elevation in insulin secretion. Therefore, GPR120 is anticipated to be the potential drug target for diabetes and obesity. Currently, a novel agonist NCG21 is being developed by researchers. It functions by activating GPR 120 and it also enhances GLP-1 release in cells. Other isoform are GPR40 and GPR119. GPR119 receptor agonist successfully brings about an upsurge in the intracellular cAMP levels, stimulating insulin synthesis and consecutively inhibiting of glucagon secretion..$^{23}$

Glucose transporter type 4 (GLUT4)-GLUT4 belongs to the family of solute carriers and brings about insulin mediated translocation of glucose across the plasma membrane. It plays a vital role in the insulin resistance progression. Hence, GLUT4 gene is a key player in maintaining glucose homeostasis in the body. Genetic mutations in the GLUT4 gene also have been associated with T2D. Therefore, it is gaining increasing interest as a therapeutic target against T2D. ${ }^{24}$

NFKB pathway-NFKB regulates the expression several genes, including growth factors, pro inflammatory cytokines, and cell adhesion molecules and plays a key role in conduction of immune responses, inflammatory responses and onset of apoptosis. ${ }^{25}$ Studies in bovine endothelial cell lines demonstrated that under hyper glycaemic conditions, production of intracellular ROS stimulates NFKB. Activation of NFKB can also be triggered by high levels of FFA's and pro-inflammatory cytokines like TNF $\alpha$. Up-regulation of these pathways results in impaired insulin action and further oxidative stress-induced insulin resistance. ${ }^{26}$ When patients with diabetes were treated with the antioxidant lipoic acid (LA), a significant suppression of NFKB activation was observed. ${ }^{27}$ These recent findings and elucidation of stress induced pathways has aided researchers to investigate and identify novel molecular targets of antioxidant action, which has the ability to block activation of the NFKB pathway.

JNK/SAPK pathway-The JNKs/SAPKs genes belongs to the complex network of MAP serine/threonine protein kinases family. ${ }^{28}$ JNK/SAPK's are stress-activated kinases, activated by stress inducing stimuli like hyperglycemia, ROS, oxidative stress, pro-inflammatory cytokines, and ultraviolet irradiation. The most crucial function of $\mathrm{JNK} / \mathrm{SAPK}$ pathway is its role in mediating apoptosis. ${ }^{29}$ Blockage of the JNK/SAPK pathway by expression of dominant negative cJun increases cell survival. Similar effect was also achieved by treatment with the thiol antioxidant N-acetyl-1-cysteine (NAC) ${ }^{30}$ Further ROS generation and consequent apoptosis could be cut down by the antioxidant vitamin C. ${ }^{31}$

P38 MAPK pathway-p38 MAPK gene is activated in response to hyperglycemia under diabetic conditions. Activation of p38 MAPK also influences a large number of cellular processes including inflammation and immunity, cell growth and apoptosis, and other signalling pathways. ${ }^{32}$ In vascular smooth muscle cells, treatment with insulin $(100 \mathrm{~nm})$ and hyperglycemia $(25 \mathrm{~mm})$ for $12-24 \mathrm{~h}$ induced the activation of $\mathrm{p} 38$ MAPK. ${ }^{33}$

The main goal of therapy of T2D is to maintain euglycemia. Till date effective treatment for diabetes is one of the biggest challenges for clinicians. Alternative measures to perk up insulin action on target tissues and identification of potent lead molecules to improve insulin secretion by $\beta$-cells, are being researched upon. Researchers are evaluating the effectiveness of natural and synthetic derivatives administration on different novel protein targets. Few of the recently discovered orally active compounds that have been efficient in maintenance of glycaemic control for type 2 diabetes patients are Biguanides (Metformin), Sulphonylureas (Glimepiride), Meglitinides (Repaglinide), Glucosidase inhibitors (Acarbose, Voglibose), Thiazolidinediones (Pioglitazone), and DPP4 inhibitors. 
Sulfonylureas cause reduction of microvascular complications, while metformin reduces of microvascular as well as macrovascular risks. Majority of patients suffering from T2D undergo multiple therapies, as monotherapy takes comparatively very long time. ${ }^{34,35}$ These therapeutic agents although provide a check over hyperglycemia; causes associated side effects like weight gain, hypoglycaemia, renal failure, gastrointestinal flatulence, edema, congestive heart failure and dehydration.

\section{Few of the most recent classes of the anti-diabetic drugs are as follows}

Biguanides-Metformin (dimethylbiguanide) was the standard first-line treatment used to reduce $\mathrm{HbAlc}$ levels by hindering gluconeogenesis, which in turn cuts down the synthesis of hepatic glucose. ${ }^{36}$ If the HbAlc reduction target is not achieved after three months of metformin administration, second-line drugs like thiazolidinediones, sulfonylureas, SGLT-2 inhibitors are usually introduced into the metformin course of therapy.

Insulin secreting agents-These molecules include namely sulfonylureas and non-sulfonylureas. Sulfonylureas elevate insulin secretion by upregulating $\mathrm{Ca}^{2+}$ permeability from voltage-gated channels. Sulfonylureas show a strong affinity towards specific receptors called sulfonylurea receptor (SUR), located on the surface of the pancreatic beta cell membrane adjacent to $\mathrm{K}^{+}$channels. SUR activation leads to reduced efflux of potassium. Surge in intracellular potassium concentration creates optimum cellular depolarization to bring forth the opening of voltage-dependent $\mathrm{Ca}^{2+}$ channels. ${ }^{37}$ This eventually elevates the intracellular $\mathrm{Ca}^{2+}$ ion concentration, bringing about insulin secretion. Second generation Sulfonylureas (glipizide, gliclazide, glibenclamide, glimepiride and glibornuride) have been found to be more potent. Non-sulfonylureas work by delaying the absorption of carbohydrates from the gastrointestinal tract. ${ }^{38}$

Sodium glucose transporter inhibitor (SGLT2)-SGLT2 is a highly efficient, transporter gene expressed chiefly in the kidneys. It facilitates transportation of $90 \%$ of glucose, amino acids and vitamins that is reabsorbed in the kidney. SGLT2 inhibitors (e.g. Dapagliflozin) obstruct the reabsorption of filtered glucose leading to glucosuria. This improves the glycaemic control and assists in calories reduction. ${ }^{39}$

Alpha-glucosidase inhibitors-This class of drugs (Acarbose, voglibose and miglitol) acts by slowing the carbohydrates absorption by inhibition of alpha-glucosidases, dropping the postprandial rise in blood glucose level. ${ }^{40,41}$

Stem cells therapy a new avenue for treatment of T2DM-Stem cells have enormous potential to divide and differentiate into various different types of specialized body. Stem cells can provide a nonexhausting source of the degenerated $\beta$ cells in T2D patients. ${ }^{42}$ There are two major faults associated with T2D, impairment of insulin function and $\beta$ cell dysfunction. $\beta$-cells can be cultivated from stem cells and further transplanted in diabetic patients. Researchers are trying to develop and establish functional $\beta$ cell mass from different types of somatic stem cells, which can prove to be a miraculous therapy. Barclay et al. ${ }^{43}$ studied Human Embryonic Stem Cells (HESCs) successfully, to be used in combination with oral therapeutic drugs like metformin/sitagliptin to treat T2D in mice. ${ }^{43}$ D'Amour et $\mathrm{al}^{44}$ developed insulin, glucagon, somatostatin, pancreatic polypeptide secreting pancreatic endocrine cells from HESCs. ${ }^{44}$ The cells differentiated in a way to imitate the in vivo development process of the islet cells. Mesenchymal Stem Cells (MSCs) obtained from various body tissues, e.g. bone marrow, adipose tissue, umbilical cord or its blood, fibroblasts and liver cells; can be effectively used to produce glucose induced insulin-secreting cells, arrest insulin resistance, recovery of liver and pancreas damage and to cure diabetic ulcers and limb ischemia. ${ }^{45,46}$ MSCs transplantation also leads to elevation of GLUT4, insulin receptor substrate 1 (IRS-1) and Akt (protein kinase B). ${ }^{47}$ Multiple intravenous transplantations need to be carried out to effectively maintain normal glucose levels and ameliorate the damaging effects of hyperglycaemia. ${ }^{48}$ Limitations of the use of stem cells are the ethical issues involved. Safety issues involved must be considered prior to clinical application.

Recent therapies in practice and pipeline drugs-Presently, doctors include basal insulin to metformin monotherapy, if HbAlc levels remain unchanged after a certain time period. Diabetes patients are also subjected to triple therapy and to additional complex injectable regimens if dual therapies are of not much help. ${ }^{49}$ The Food and Drug Administration (FDA) approved lixisenatide (Adlyxin, Sanofi), a once-daily at mealtime GLP-1R agonist injection, as an add-on to diet and exercise for the treatment of adults with T2D, in July, $2016 .{ }^{50}$ Clinical trials performed with this drug effectively met the criteria of $\mathrm{HbAlc}$ reductions. Few adverse events included nausea, hypoglycaemia, and vomiting. Another promising candidate drugs in pipeline is Ertugliflozin (Pfizer), an oral, once-daily SGLT-2 inhibitor which demonstrated statistically significant $\mathrm{HbA} 1 \mathrm{c}$ reductions T2D subjects. ${ }^{51}$ Ertugliflozin is rapidly absorbed and acts by lowering blood glucose levels by causing the kidneys to remove glucose from the body through the urine. Ertugliflozin is eliminated quickly from the body by glucuronidation..$^{52}$ Though, ertugliflozin has been proposed to be used in combination with other drugs namely Merck manufactured Sitagliptin, however Ertugliflozin if approved will face hard competition with the already well-established SGLT-2 inhibitors. The probable marketing date for ertugliflozin is by the end of 2017. Currently, Novo Nordisk is formulating (ultra-rapid/faster acting insulin aspart), intended to match to the physiological profile of prandial insulin and to enhance the delivery of insulin required post meal. ${ }^{53}$ Once approval by the FDA anticipated by end of 2017, FIAsp would be available in the FlexTouch prefilled delivery syringe and a $10-\mathrm{mL}$ vial. ${ }^{54}$ Simultaneously, FDA has accepted NDA filing for the drug ITCA 650 (continuous subcutaneous delivery of exenatide) formulated by Intarcia Therapeutics on February 3, 2017. Exenatide is a GLP-1R agonist, currently being marketed as twice-daily/onceweekly self injections. ${ }^{55}$ The drug therapy is designed to utilize a subcutaneous osmotic mini-pump (employing Intarcia's innovative technology platform, the Medici Drug Delivery System) for continuous delivery of exenatide drug for a period of one year. The FREEDOM clinical trial program conducted for ITCA 650 demonstrated highest $\mathrm{HbAlc}$ reductions in patients receiving combination dose of metformin. ${ }^{56}$ ITCA 650 is the first once/twice yearly, injection-free GLP-1 therapy available globally. ${ }^{57}$ Semaglutide by Novo Nordisk is also being developed as a once-weekly subcutaneous injection for patients with T2D. In the SUSTAIN 3 clinical trial, semaglutide $(1.0 \mathrm{mg})$ administered once weekly showed significant enhancement in glycemic status when compared with extended-release exenatide $(2.0 \mathrm{mg}))^{58}$ Another potent drug OG217SC (Novo Nordisk) is being developed as a once daily tablet formulation, with an absorption enhancing excipient called sodium N-(8-[2-hydroxybenzoyl] amino) caprylate. On FDA approval its anticipated launch date is 2020 and it is expected to be a blockbuster drug as all other GLP-1R agonists 
are injectables. OG217SC and ITCA 650 might provide a tough competition to each other and seem to hold promising future in T2D treatment, although OG217SC will likely have an upper hand due to ease of administration..$^{53}$ The market acceptability and wide spread acceptance by patient population are two determining factors that decide the fate of a drug, however, evidences from high quality clinical trial is essential to be established for these compound for determining the safety and efficacy profiles. We believe that it is not one drug but multiple drugs will be needed to cater to the various intricacies associated with T2D being a disease of epigenetic nature in different population across the globe.

\section{Conclusion}

Extensive research is ongoing to generate novel outlook into of mechanistic understanding of hyperglycemia and designate potent pharmacological targets for the treatment of insulin resistance and beta cell dysfunction. The first discovered oral anti diabetic drug sulfonylurea had several off target effects. Phenformin was pulled off the market due to its adverse effects, whereas Metformin comparatively showed improved regulation of high blood sugar level, and did not cause any lethal side effects. ${ }^{59}$ Later on thiazolidinediones were discovered followed by Solute carriers GLUT1-4 and SGLT2 which were not explored earlier as therapeutic drug targets for T2D. Presently, more promising novel drug candidates are those which act as agonists for DPP4 inhibitors, SGLT2, GPR120, G-protein-coupled receptors and peroxisome proliferator-activated receptor. Although side effects associated with various synthetic drugs have renewed the interest in traditional medicinal plants and herbal preparations to derive treatment of diabetes, still the novel candidates have a promising usage in treating T2D.

Presently the T2D market is populated with several kinds of drug formulations, but they tend to lose their efficacy after a certain period of time. Generally T2D patients are administered multiple therapy to control their glycaemic levels, as monotherapy are not solely effective. Also, these treatment regimes do not cure the complications permanently and cause several side effects additionally. Hence, once glycaemic control is achieved, novel molecules targeted against T2D should essentially be effective in prevention of cardiovascular events by reduction of blood pressure, cutting down body weight, triglycerides and low-density lipoprotein cholesterol reduction. Also, advent of new therapies should focus on non-peptide drugs which directly activate the insulin receptors. Simultaneously, advancements the in stem cell therapy would also provide an improved and permanent solution to combat T2D. Stem cell therapy does not encounter technical issues like graft rejection and dearth of donors, unlike organ transplantations as patients' self stem cells are differentiated in vitro to islet cells. Hence, the novel techniques and synthetic leads expected to be launched in the upcoming 10 years may have the potential to transform T2D treatment regime and render an improved glycaemia control status and weight loss when compared with ongoing traditional treatments.

\section{Acknowledgements}

None.

\section{Conflict of interest}

The author declares no conflict of interest.

\section{References}

1. International diabetes federation. The Diabetes Atlas. 6th ed. Africa at a Glance; 2013.

2. World health organization. Diabetes. Fact sheet $N^{\circ} 312 ; 2013$.

3. Liu Q, Chen L, Hu L, et al. Small molecules from natural sources, targeting signaling pathways in diabetes. Biochim Biophys Acta. 2010;1799(1012):854-865.

4. Randle J, Garland B, Hales N, et al. The glucose fattyacid cycle. Its role in insulin sensitivity and the metabolic disturbances of diabetes mellitus. Lancet. 1963;1(7285):785-789.

5. Shetty P. Epigenetics and lifestyle are conspiring to inflict a massive epidemic of type 2 diabetes in the subcontinent. Nature. 2012;485:S14-16.

6. Johnson AMF, Olefsky JM. The origins and drivers of insulin resistance. Cell. 2013;152(4):673-684.

7. Porte D, Kahn E. Mechanisms for hyperglycemia in type II diabetes mellitus: therapeutic implications for sulfonylurea treatment-an update. Am J Med. 1991;90(6A):8S-14S.

8. Marles RJ, Farnsworth NR. Antidiabetic plants and their active constituents. Phytomedicine. 1995;2(2):137-189.

9. Schinner S, Scherbaum WA, Bornstein SR, et al. Molecular mechanisms of insulin resistance. Diabet Med. 2005;22(6):674-682.

10. Hotamisligil S. Molecular mechanisms of insulin resistance and the role of the adipocyte. Int J Obes Relat Metab Disord. 2000;24(4):S23-27.

11. Brownlee M. Biochemistry and molecular cell biology of diabetic complications. Nature. 2001;414(6865):813-820.

12. Turner RC, Holman RR, Stratton IM, et al. Effect of intensive bloodglucose control with metformin on complications in overweight patients with type 2 diabetes (UKPDS 34). Lancet. 1998;352(9131):854-865.

13. Maxwell SRJ, Thomason H, Sandler D, et al. Antioxidant status in patients with uncomplicated insulin-dependent and non-insulin-dependent diabetes mellitus. Eur J Clin Invest. 1997;27(6):484-490.

14. Uchimura K, Nagasaka A, Hayashi R, et al. Changes in superoxide dismutase activities and concentrations and myeloperoxidase activities in leukocytes from patients with diabetes mellitus. J Diabetes Complications. 1999;13(5-6):264-270.

15. Allen RG, Tresini M. Oxidative stress and gene regulation. Free Radic Biol Med. 2000;28(3):463-499.

16. Melov S. Mito chondrial oxidative stress. Physiologic consequences and potential for a role in aging. Ann NY Acad Sci. 2000;908:219-225.

17. Bursell SE, Clermont AC, Aiello LP, et al. High-dose vitamin E supple mentation normalizes retinal blood flow and creatinine clearance in patients with type 1 diabetes. Diabetes Care. 1999;22(8):1245-1251.

18. Rieusset J, Andreelli F, Auboeuf D, et al. Insulin acutely regulates the expression of the peroxisome proliferator-activated receptor-gamma in human adipocytes. Diabetes. 1999;48:699-705.

19. Balasubramanyam M, Mohan V. Current concepts of PPAR- $\gamma$ signalling in diabetes mellitus. Current Science. 2000;79(10):1440-1446.

20. Shravanti K, Kumar PK, Raju MB, et al. A Review on structure based drug design of protein tyrosine phosphatase $1 \mathrm{~B}$ inhibitors for target for obesity and type 2 diabetes mellitus. J Pharma Res. 2010;3(12):2939-2940.

21. Goldstein J. Protein-tyrosine phosphatase 1B (PTP1B): a novel therapeutic target for type 2 diabetes mellitus, obesity and related states of insulin resistance. Curr Drug Targets Immune Endocr Metabol Disord. 2001;1(3):265-275. 
22. Fredriksson R, Höglund J, Gloriam E, et al. Seven evolutionarily conserved human rhodopsin $\mathrm{G}$ protein-coupled receptors lacking close relatives. FEBS Lett. 2003;554(3):381-388.

23. Huang S, Czech M. The GLUT4 glucose transporter. Cell Metab. 2007;5(4):237-252.

24. Karin M, Ben Neriah Y. Phosphorylation meets ubiquitination: the contro of NFKB activity. Annu Rev Immunol. 2000;18:621-663.

25. Evans JL, Goldfine ID, Maddux BA, et al. Oxidative stress and stress activated signaling pathways: a unifying hypothesis of type 2 diabetes. Endocr Rev. 2002;23(5):599-622.

26. Hofmann A, Schiekofer S, Kanitz M, et al. Insufficient glycemic control increases nuclear factor-B binding activity in peripheral blood mononuclear cells isolated from patients with type 1 diabetes. Diabetes Care. 1998;21(18):1310-1316.

27. Pages G, Berra E, Milanini J, et al. Stress-activated protein kinases (JNK and $\mathrm{p} 38 / \mathrm{HOG}$ ) are essential for vascular endothelial growth factor mRNA stability. J Biol Chem. 2000;275(34):26484-26491.

28. Basu S, Kolesnick R. Stress signals for apoptosis: ceramide and c-Jun kinase. Oncogene. 1998;17(25):3277-3285.

29. Xia Z, Dickens M, Raingeaud J, et al. Opposing effects of ERK and JNK-p38 MAP kinases on apoptosis. Science. 1995;270(5240):13261331.

30. Ho M, Liu H, Liau S, et al. High glucose-induced apoptosis in human endothelial cells is mediated by sequential activations of c-Jun $\mathrm{NH}(2)$ terminal kinase and caspase-3. Circulation. 2000;101(22):2618-2624.

31. Lewis S, Shapiro S, Ahn G. Signal transduction through MAP kinase cascades. Woude GFV, et al. editors. Adv Cancer Res. San Diego, USA: Academic Press Inc; 1998;74:49-139.

32. Obata T, Brown E, Yaffe B. MAP kinase pathways activated by stress: the p38 MAPK pathway. Crit Care Med. 2000;28(4):N67-77.

33. Igarashi M, Wakasaki H, Takahara N, et al. Glucose or diabetes activates p38 mitogen-activated protein kinase via dif- ferent pathways. J Clin Invest. 1999;103(2):185-195.

34. Inzucchi SE. Oral antihyperglycemic therapy for type 2 diabetes: scientific review. JAMA. 2002;287(3):360-372.

35. Turner C, Cull A, Frighi V, et al. Glycemic control with diet, sulfonylurea, metformin, or insulin in patients with type 2 diabetes mellitus: progressive requirement for multiple therapies (UKPDS 49). UK prospective diabetes study (UKPDS) Group. JAMA. 1999;281(21):2005-2012.

36. Pernicova I, Korbonits M. Metformin-mode of action and clinical implications for diabetes and cancer. Nature Rev Endocrinol. 2014;10(3):143-156.

37. Nguyen NDT, Le LT. Targeted proteins for diabetes drug design. Adv Nat Sci Nanosci Nanotechnol. 2012;3:1-9.

38. Henquin C. Pathways in beta-cell stimulus-secretion coupling as targets for therapeutic insulin secretagogues. Diabetes. 2004;53(3):S48-58.

39. Neumiller J, White Jr, Campbell K. Sodium-glucose co-transport inhibitors: progress and therapeutic potential in type 2 diabetes mellitus. Drugs. 2010;70(4):377-385.

40. Lebovitz E. Alpha-Glucosidase inhibitors. Endocrinol Metab Clin North Am. 1997;26(3):539-551.
41. El-Badri N, Ghoneim A. Mesenchymal stem cell therapy in diabetes mellitus: progress and challenges. J Nucleic Acids. 2013;2013:194858.

42. Kim JJ, Kido Y, Scherer E, et al. Analysis of compensatory beta-cell response in mice with combined mutations of Insr and Irs2. Am J Physiol Endocrinol Metab. 2007;292(6):E1694-1701.

43. Barclay S. Combination of stem cell and drug therapy could reverse type 2 diabetes. Health line Media; 2015

44. D'Amour A, Bang G, Eliazer S, et al. Production of pancreatic hormoneexpressing endocrine cells from human embryonic stem cells. Nat Biotechnol. 2006;24(11):1392-1401.

45. Jiang Y, Jahagirdar N, Reinhardt L, et al. Pluripotency of mesenchymal stem cells derived from adult marrow. Nature. 2002;418(6893):41-49.

46. Wang L, Zhao S, Mao H, et al. Autologous bone marrow stem cell transplantation for the treatment of type 2 diabetes mellitus. Chin Med J (Engl). 2011;124(22):3622-3628.

47. Si Y, Zhao Y, Hao H, et al. Infusion of mesenchymal stem cells ameliorates hyperglycemia in type 2 diabetic rats: identification of a novel role in improving insulin sensitivity. Diabetes. 2012;61(6):1616-1625.

48. Hao H, Liu J, Shen J, et al. Multiple intravenous infusions of bone marrow mesenchymal stem cells reverse hyperglycemia in experimental type 2 diabetes rats. Biochem Biophys Res Commun. 2013;436(3):418-423.

49. American Diabetes Association. Standards of medical care in diabetes-2015. Diabetes Care. 2015;38(1):S1-90.

50. Sanofi. Sanofi receives FDA approval of Adlyxin for treatment of adults with type 2 diabetes. USA; 2016.

51. Pfizer. Merck and Pfizer announce two pivotal phase 3 studies for ertugliflozin, an investigational SGLT-2 inhibitor, met primary endpoints, showing significant A1C reductions in patients with type 2 diabetes; 2016

52. Miao Z, Nucci G, Amin N, et al. Pharmacokinetics, metabolism, and excretion of the antidiabetic agent ertugliflozin (PF 04971729) in healthy male subjects. Drug Metab Dispos. 2013;41(2):445-456.

53. Cuaron J, Gburcik V, Prabhakar B. PharmaPoint: Type 2 Diabetes-Global Drug Forecast and Market Analysis to 2025. New York, USA: Global Data; 2016.

54. Novo Nordisk. Novo Nordisk files for regulatory approval of "fasteracting insulin aspart" in the US; 2015.

55. Intarcia Therapeutics, ITCA 650; 2016.

56. Intarcia Therapeutics. Intarcia announces two positive phase 3 trials for ITCA 650 in type 2 diabetes: FREE FREEDOM-1 and FREEDOM-1 High Baseline (HBL) study results; 2014.

57. Intarcia Therapeutics. Intarcia presents FREEDOM-2 trial results in type 2 diabetes demonstrating clinically meaningful superiority and sustained glucose control and weight reduction for ITCA 650 versus Januvia: oral presentation at ADA 76th scientific sessions; 2016.

58. Novo Nordisk. Semaglutide demonstrated superior improvements in glycaemic control vs sitagliptin (SUSTAIN 2) and exenatide ER (SUSTAIN $3)$ in two clinical trials in adults with type 2 diabetes. USA; 2016.

59. Bolen S, Feldman L, Vassy J, et al. Systematic review: comparative effectiveness and safety of oral medications for type 2 diabetes mellitus. Ann Intern Med. 2007;147:386-399. 